\title{
SERVICE-ORIENTED CONCEPT OF A HOLONIC ENTERPRISE - ENABLING ADAPTIVE NETWORKS ALONG THE VALUE CHAIN
}

\author{
Georg Weichhart, Alexander Hämmerle, Kurt Fessl \\ PROFACTOR Produktionsforschungs GmbH, Wehrgrabengasse 1-5, A-4400 Steyr \\ \{Georg.Weichhart |Alexander.Haemmerle|Kurt.Fessl]@Profactor.at
}

\begin{abstract}
In this paper we are discussing a holonic architecture for a management and control software, supporting users within enterprises and networks of enterprises in their co-ordination tasks. Holons are extended with functionality identified by intelligent software agent and multi agent systems research. This improves communication capabilities, flexibility, and the overall system robustness. Furthermore we discuss the need for a service oriented approach, which enables us to let the same agent kernel, which is handling mainly coordination and communication, be applied to hierarchy levels ranging from the virtual-enterprise-level to the shop-floor level.
\end{abstract}

\section{INTRODUCTION}

"Recently the manufacturing industry is facing a continuous change from a supplier's market to a customer's market" (Bussmann 1999). Customers are asking for complex and personalized products. The first demand adds to the complexity, that the production machinery needs to handle. This wouldn't be too much of a problem, if the second wouldn't imply that the batch size is decreased to one in the worst case. Therefore the time to set-up a new product version has to be reduced towards zero, at almost no cost. "These effects can be summarized as increasing complexity and continuous change at no cost" (Bussmann 1999).

\subsection{Alternate Approaches}

Traditional control and management approaches are optimistic. A plan is created, where each unit follows the optimal way through the system. There is one implied precondition: Everything is working correct.

Even if only a small degree of complexity is reached, but there is only one way through the system, it can been proven that it is almost impossible to guarantee a working system:

If two or more independent systems have to be used one after the other, the probability that the whole system functions can be calculated by multiplying the sub-systems probability to function $(\mathrm{P}(\mathrm{A}$ and $\mathrm{B})=\mathrm{P}(\mathrm{A}) \cdot \mathrm{P}(\mathrm{B}))$. For the following calculations we assume to need a $99 \%$ probability of the whole system working: 
Table 1 - Needed availability of subsystems

\begin{tabular}{|c|l|}
\hline $\begin{array}{c}\text { Number of } \\
\text { subsystems }\end{array}$ & $\begin{array}{l}\text { Necessary probability of subsystem to work has to be at } \\
\text { least }\end{array}$ \\
\hline 2 & 0,9949874371 \\
\hline 20 & 0,9994976094 \\
\hline 200 & 0,9999497496 \\
\hline
\end{tabular}

The conclusion is that the probability of a malfunction of the whole system gets higher the more subsystems are involved. To fight this problem redundancy has to be introduced. Since there is more than one possible way through the system, the probability of success is higher. Redundancy allows flexibility, but increases complexity. Scheduling and Planing is getting harder because of a increased number of possible solutions and interdependencies.

To fight this complexity, different groups have conducted research on decentralized systems (see for example Weiss 1999, Walsh 1998, Tönshoff et. al. 2000 and research on Multi Agent Systems in general). In these approaches independent systems have knowledge about their local subsystem. To reach a solution that satisfies all participating sub-systems' local optimization function, intense communication has to be conducted. With decentralized approaches in general it can not always be guaranteed that a common satisfactory point can be reached. Additionally the amount of communication is high compared to centralized approaches.

Centralized systems introduce other problems. A scale-up of such a system often leads to an overload of the central unit, where most of the decisions are made. Flexibility and robustness are additional problems with centralized approaches.

We therefore use a holonic approach to balance the advantages of those two approaches (see also for different domains Ulieru et. al. 2001, Fischer 1999, Bongaerts et. al. 2000).

\subsection{Holonic and Software Agents}

Our view of a Holon is that it is a Software Agent, embedded in a recursively designed Multi-Agent-System. All the attributes identified by (Wooldridge and Jennings 1995) and (Nwana 1996), for example, will be implemented by our holons (see also Gerber and Russ 2001), by a varying extend, dependent on the system level, which the individual holon is part of.

Since holonic architectures are typically designed to work on the shop floor, on lower holarchy levels the holons will degenerate to simple agents, having less autonomy, but focus on reactiveness. The problems tackled by holons interfacing hardware are simple enough to allow a more centralized but time efficient control.

The higher the level the holon is located within the holarchy, the more intelligence is necessary to make decisions. For example the time frame considered is increased, or the number of system variables that have to be considered for a decision is higher. The tasks to be done, and the problems tackled on theses levels are complex, but on the other side do not have real time constraints as hard as on hardware close levels. It shall be possible to integrate a holon in a multi agent system where it won't be distinguishable from any other intelligent agent. 


\section{THE ARCHITECTURE}

\subsection{The Constructs (Objects) Used}

Our Software-Architecture is based on two constructs:

- the Resource-Holon and

- the Service-Object.

Resources are necessary to apply services to a (intermediate-) product according to some plan. This application of services generates additional value. Two types of resources are identified. On the one hand machines, persons and the like, that are actively applying services to products. On the other hand electrical power, cooling water, construction material and sub- products like tires consumed by the product and after being applied might not be disunited from the product without damaging it.

A service describes the process of a resource to apply some material or energy consuming operation that in the long term adds value, which in turn is sold to the world outside by finally selling the product (i.e. the enterprise gains profit).

\subsection{The Holons}

\subsubsection{General}

Basic resource holons encapsulate a piece of hardware or an external system. These holons join to form a super ordinate system (e.g. machines form a shop floor).

We refer to this system as super holon. When considering this super ordinate holon we label the building blocks sub holons. Each level, but the lowest and the highest, is formed by holons that are sub-holons when considering higher level holons, but are super-holons for lower level holons.

We do not assume anything about the internal architecture of a basic holon. A basic holon in our words is a holon, that does not consist of sub-holons.

Several super-holons formed by basic holons will join again to reach a common goal. The recursive architecture leads to a holarchy, where holons are formed over several levels.

The formation may happen dynamically and temporary. Each holon might provide its services to more than one super-holon (see for example Wyns 1999, Christensen 1994). The offered services might be clusters of services offered by subholons, atomic services, and, of course, a combination of those possibilities.

Service clusters are used to reduce complexity. We do not define the methods how the sub services are clustered, or even force a holon to cluster the services of it's sub-holons. In a worst case scenario the Holon might offer all possible permutations of sub-services as separate clusters.

The resource-holons in this architecture are autonomous. They decide on their own which services when to offer. However an efficient way to influence decisions of sub-holons, especially on the lower levels is needed. These influences could be based on parameters like priority and cost, which are provided by holons that have better knowledge of the overall system (i.e. the global objective function).

The following picture gives an overview of our architecture: 


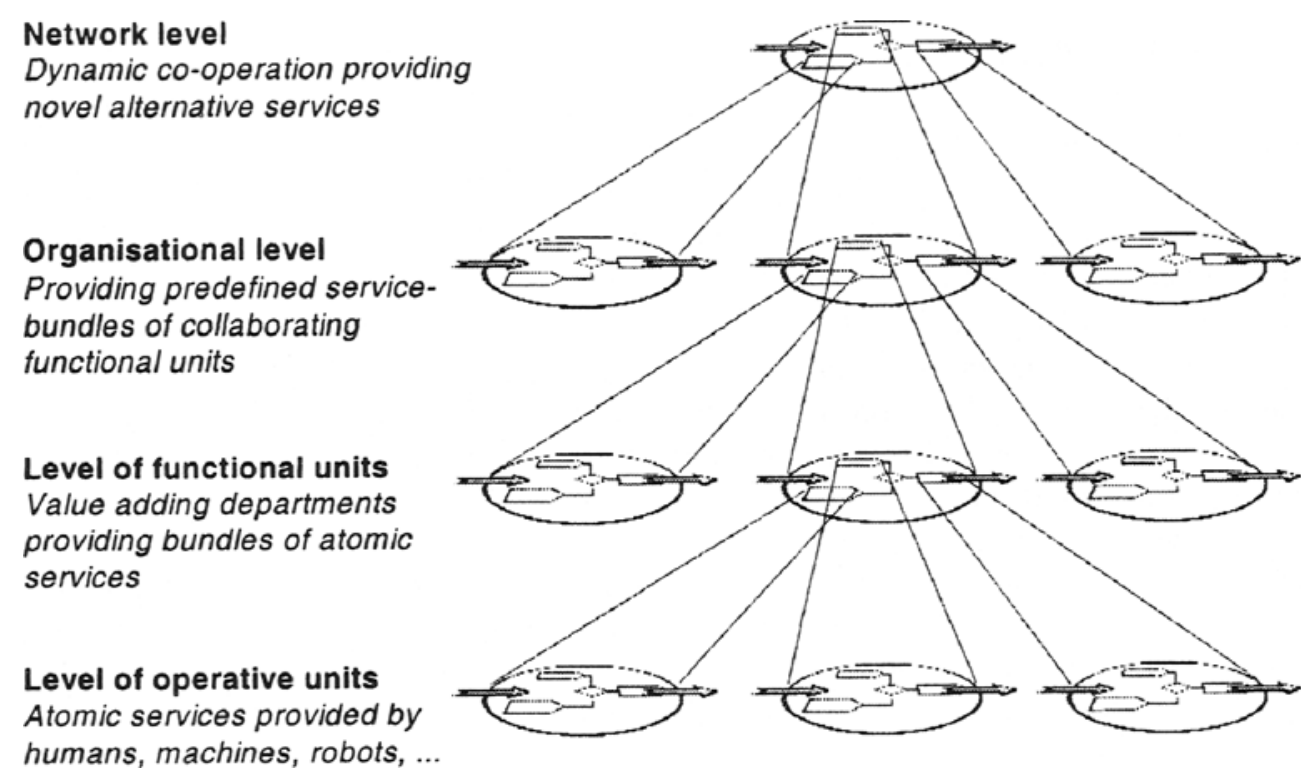

Figure 1. Holarchy levels

Holons on the network level are formed dynamically and temporary. The resource holons on the organisational level are rather longer lasting systems. On the levels below, time-consuming decisions are reduced, and it is gradually focused on runtime efficiency. Despite this picture might suggest a strict hierarchy, this is not the case. Each holon may be part of multiple holons.

\subsubsection{Identification of Basic Holons}

Recently research has been carried out to create a methodology that supports system designer in identifying Software Agents (see Wooldridge and Ciancarini 2000 for an overview). As recognised by (Bussmann et. al. 2000) "none of these methodologies is applicable to the design of agent-based production control systems; they either provide analysis models that are inappropriate for production control or else they lack comprehensive design rationales".

In our architecture, the physical objects (e.g. machines, work-pieces) are represented by holons. This is possible, since, our architecture allows a gradually degeneration from intelligent agents to eventually even passive objects. The services provided depend on the possibilities and permissions the hardware has. Theses basic holons will join and form higher level holons with a dedicated mission (e.g. shop floor). Our service oriented architecture is flexible enough to blur the borders between real-time requirements and (time-consuming) intelligence, and allow a gradually introduction of intelligent agents. It also allows to implement loadbalancing algorithms as described later.

\subsubsection{Basic Types of Holons}

Guided by the basic PROSA architecture (Wyns 1996), we identified the following types of holons in a production enterprise: 1) Order-holon, 2) Product-holon, 3) 
Resource-holon (e.g. Enterprise-holon, Shop-Floor-Holon, Machine-holon, RawMaterial-Holon, Energy-Holon), 4) Work-piece-holon.

The following use case shall demonstrate the knowledge and possibilities possessed by each:

A customer request triggers the generation of an Order-Holon. This holon contacts the "Yellow Pages" Service of the virtual. It looks up standard products directly and additionally all the services (processes) necessary to build the desired product. A compatible product-holon is found. This holon provides information services about the represented product. The product-holon might offer an ontology describing in detail which services are applied. If this process is considered to be of competitive advantage for the providing enterprise, this holon will interactively decide what information to provide.

The enterprise-holons known by the product holon are contacted. Eventually an auction is held to sell the order (the interaction protocol has to be agreed by the participating holons and is not predetermined by our architecture). However, the order-holon finally contacts one enterprise holon able to produce the desired product. Depending on the interaction protocol, the price, time of delivery, and quality might be already negotiated, or this is done now with user support.

The winning enterprise-holon contacts the holon able to provide internal coordination services necessary to organize the production process. The order-holon is not allowed to get into the enterprise. This would weaken the encapsulation, and introduce security risks. Instead an internal representation, a work-piece-holon, is started. Additionally to the knowledge it inherits from the order-holon, it has detailed information about the production process. This holon mainly provides information services about its current state, and the resources it has used. It might consist of intermediate products, or parts (tires of a car for example), and is therefore also organized as holon. These work-piece-holons contact the machines necessary to apply the desired services, according to the process-description. The sub-holons of the work-piece-holon communicate with different resource-holons to co-ordinate the production process.

The machine-holons subcontract other resource-holons if necessary. We do not address scheduling and other problems raised on the shop-floor here. In our architecture these are services needed by the shop-floor holon.

\subsection{The Services}

\subsubsection{Services, Ontologies and the Product-Holon}

The provision of services is the possibility of enterprises to generate value. We consider a product for example as the aggregation of several services (cluster of services).

Service objects are not holons or agents because the lack of autonomy and intelligence. For efficiency reasons they should be as lightweight as possible.

Resource-holons decide which services (or service clusters) to provide to its peers. The distinction between a service and a service cluster is only possible by checking against the according ontology. It shall not be determinable by just querying the service-object. Because each holon encapsulates its sub-holon it is possible that each holon might have its own ontology. For efficiency reasons one 
single centralised ontology, which is referred to by each holon, is preferable at least within an enterprise or even better within the enterprise network.

Product-holons represent the products as described by the ontology to the holons outside the originating enterprise. This holon has a detailed description of the services needed, and the order in which they will be applied (i.e. the productionprocess). This encapsulation of the ontology by the product-holon makes it possible to determine at runtime the extend to which the production process is visible to a specific order-holon.

In addition to the above mentioned value generating services other (sub-) services are necessary. These services are information, communication and coordination services, necessary for internal use of the system. These services do not generate an addition value by them self.

The demanded robustness of the systems needs co-ordination mechanisms to allow plugging in of new resources (hardware) at any time. Additionally a breakdown of the hardware needs to be communicated to other holons by either logging off the whole machine or not providing the affected services anymore. Since a breakdown eventually implies that the holon has no change to logoff, control strategies are necessary.

\subsubsection{Value generating Services}

Depending on the task to be fulfilled, or product offered, the resource-holons provide different services. A drilling machine may provide the service "Drill hole with a diameter of $\mathrm{X} \mathrm{mm".} \mathrm{A} \mathrm{cooling-water-holon} \mathrm{might} \mathrm{offer} \mathrm{the} \mathrm{service} \mathrm{"Provide}$ cooling-water in quality $\mathrm{A}$ ".

The work-piece holon has knowledge about the production process. To apply the services according to the process description, the resources and the application of their services have to be coordinated.

\subsubsection{Co-ordination Services}

Each resource holon needs co-ordination services to co-ordinate its internal subholons, and to present the internal services to the super system (gateway functionality).

These services are often jointly provided by a mediator agent or holonhead (e.g. Haemmerle 2002, Shen et al 2000) in other architectures. We don't use a Mediator, any holon able to do so may offer co-ordination services. This means that internal co-ordination and gateway functionality could be provided by different holons. The service-oriented approach allows to implement load-balancing and fallback algorithms. At runtime a resource will identify dynamically whose service to use.

The decision might be based on attributes like workload of individual holons. For a fallback support, to have the system stable even if the co-ordinator goes offline, duplication of co-ordination information is necessary. The primary coordinator duplicates its "database". If it fails, the backup co-ordinator will be promoted to be the primary co-ordinator. The same holds true for other types of services.

Necessary co-ordination services are yellow-pages and white-pages services. These are needed to contact the right holons if a special type of service is needed. 
Another required set of services allows plug and play capabilities. A standard login-process needs to be designed to automate the process of entering (joining) a holon and publish the new available services in the white- and yellow-pages. If new services are introduced by the entering resource they might also be communicated (i.e. offered) to the outside.

The encapsulation of the sub-holons in one holon (the super holon) guarantees that the addition or subtraction of some holon has no direct impact on the peers of that (super-)holon. Holons might experience a difference only if a type of service is not available any more or a new one is available.

\subsubsection{Information Services and Advanced Decision Support}

When stepping up the holarchy more and more holons are clustered indirectly (i.e. more and mores system variables are introduced). Therefore the amount of data available and probably needed for a decision is increased. The clustering of offered services will help to decrease the level of detail. Data Mining algorithms may be implemented utilising the information services provided by sub-holons.

The service concept allows the ease introduction of heuristic optimisation functions. Depending on parameters like priority or available system resources, different more or less resource consuming algorithms might be chosen.

\section{CONCLUSION}

We have proposed an architecture that allows to control and automate processes within the whole enterprise and even dynamic networks of enterprises. The servicecentered approach allows flexibility and robustness. Contrary to other architectures, co-ordination and information services are not provided by a single mediator. This allows the easy implementation of load balancing and fallback algorithms, which adds to robustness.

The fine granularity that is provided by the services centered approach is to be used to gradually decrease the intelligence, and increase time-efficiency on lower enterprise levels. A broad spectrum of different implementations is made possible.

\section{ACKNOWLEDGMENTS}

The authors are grateful for financial contributions of the VPTÖ, the Austrian association for promoting manufacturing sciences

\section{REFERENCES}

1. Sivaram Balasubramanian, Robert W. Brennan, Douglas H. Norrie, "An architecture for metamorphic control of holonic manufacturing systems”, Computers in Industry 46, 2001.

2. Luc Bongaerts, László Monostori, Duncan McFarlane, Botond Kádár, "Hierarchy in distributed shop foor control", Computers in Industry 43, Elsevier, 2000.

3. Stefan Bussmann, Duncan C. McFarlane, "Rationales for Holonic Manufacturing Control", Proceedings of 2nd International Workshop on IMS, Leuven, Belgium, 1999. 
4. Stefan Bussmann “An Agent-Oriented Architecture for Holonic Manufacturing Control”, First Open Workshop IMS Europe, Lausanne Switzerland, 1998.

5. James H. Christensen, "Holonic Manufacturing Systems: Initial Architecture and Standards Directions”, First European Conference on HMS, Hannover, Germany, 1994.

6. Klaus Fischer, "Agent-Based Design of Holonic Manufacturing Systems", Journal of Robotics and Autonomous Systems 27, Elsevier Science, 1999.

7. Andreas Gerber, Christian Russ, "A Holonic multi-agent Infrastructure for Electronic Procurement”, Proceedings of the 14th International FLAIRS Conference, 2001.

8. Alexander Haemmerle, Kurt Fessl, Georg Weichhart "The MaBE Project: An Agent-Based Environment for Business Networks", submitted to HOLOMAS, 2002.

9. Hyacinth S. Nwana, "Software Agents: An Overview", Knowledge Engineering Review, Vol. 11, No 3, Cambridge University Press, 1996.

10. Weiming Shen, Francisco Maturana, and Douglas H. Norrie "MetaMorph II: an agent-based architecture for distributed intelligent design and manufacturing", Journal of Intelligent Manufacturing, Kluwer Academic Publishers, 2000

11. Weiming Shen, Douglas H. Norrie, Jean-Paul A. Barthès, "Multi-Agent Systems for Concurrent Intellignet Design and Manufacturing", Taylor and Francis, London, New York, 2001.

12. H.-K. Tönshoff, I. Seilonen, G. Teunis, P.Leitao, "A Mediator-Based Approach For Decentralised Production Planning, Scheduling, And Monitoring", Proceedings of CIRP International Seminar on Intelligent Computation in Manufacturing Engineering (ICME), Italy, 2000.

13. Mihaela Ulieru, Scott S. Walker, and Robert W. Brennan, "The Holonic Enterprise as a Collaborative Information Ecosystem", Proceedings of the Workshop on Holons: Autonomous and Cooperating Agents for Industry, Autonomous Agents 2001, Montreal, May 29, 2001

14. Paul Valckenaers, Henrik Van Brussel, Jo Wyns, Luc Bongaerts, Patrick Peeters, "Desining Holonic manufacturing systems", Robotics and Computer-Integrated Manufactruing 14, 1998.

15. William E. Walsh, Michael P. Wellmann, "A Market Protocol for Decentralized Task Allocation", Extended version of a paper in Proceedings of the $3^{\text {rd }}$ ICMAS, Paris, France, July 1998.

16. Gerhard Weiss (editor), "Multiagent systems: a modern approach to distributed artificial intelligence", Massachusetts Institute of Technology, 1999

17. Michael Wooldridge and Nicholas R. Jennings, "Intelligent Agents: Theory and Practice", Knowledge Engineering Review Volume 10 No 2, Cambridge University Press, 1995.

18. Michael Wooldridge and Paolo Ciancarini, "Agent-Oriented Software Engineering: The State of the Art”, Agent-Oriented Software Engineering, Paolo Ciancarini, Michael J. Wooldridge (editors), Lecture Notes in Computer Science Vol. 1957, Springer 2000

19. Joe Wyns, "Reference Architecture for Holonic Manufacturing Systems", Phd. Thesis, Katholieke Universiteit Leuven Faculteit Toegepaste Wetenschappen, Arenbergkasteel, B-3001 Heverlee (Belgium), 1999 